\title{
Pleuropulmonary blastoma in an adult: A case report
}

\author{
Nidhi Paliwal, Kumud Gupta, Shalini Mullick, \\ Ravindra K Dewan, Sandeep Katiyar
}

\begin{abstract}
Introduction: Pleuropulmonary blastoma (PPB) is a dysontogenetic neoplasm of childhood that appears as a pulmonary and/or pleural-based mass. PPB is of three types: cystic (type I), mixed (type II), or solid (type III). It is rarely observed in adults with only a few cases reported in literature. Case Report: We present a case of a 30-year-old male with complaints of chest pain, dyspnea and low grade fever. His computed tompgraphy scan of chest showed three multicystic lesions in right hemithorax. Cytomorphological examination of imprint smears of biopsies taken from the masses suggested malignant lesion and on histological examination, diagnosis of pleuropulmonary blastoma type II was made. Conclusion: Pleuropulmonary blastoma is a rare and aggressive malignant tumor of childhood. Familial disposition and association of PPB tumor with other childhood malignancies has been suggested in literature. Pathologically, the tumor tissue is composed of primitive blastemal
\end{abstract}

Nidhi Paliwal ${ }^{1}$, Kumud Gupta ${ }^{2}$, Shalini Mullick ${ }^{3}$, Ravindra K Dewan ${ }^{4}$, Sandeep Katiyar ${ }^{5}$

Affiliations: ${ }^{1}$ Senior Resident, Pathology, LRS Institute of TB \& Respiratory Diseases, New Delhi, India; ${ }^{2} \mathrm{Head}$, Pathology, LRS Institute of TB \& Respiratory Diseases, New Delhi, India; ${ }^{2} \mathrm{DNB}$, Specialist, Pathology, LRS Institute of TB \& Respiratory Diseases, New Delhi, India; ${ }^{4}$ Head, Thoracic Surgery, LRS Institute of TB \& Respiratory Diseases, New Delhi, India; ${ }^{5}$ Resident, Respiratory Medicine, LRS Institute of TB \& Respiratory Diseases, New Delhi, India.

Corresponding Author: Nidhi Paliwal, Quarter no. 1053, Sector-8, R K Puram, New Delhi, Delhi, India - 110022; Ph: 91-9999857039; Fax: 91-26568227; Email: npaliwal1983@gmail.com

Received: 07 July 2012

Accepted: 24 September 2012

Published: 01 January 2013 cells with focal areas of malignant mesenchyme. Therapy should include surgical tumor resection and subsequent chemotherapy and radiotherapy. PPB is very rare tumor in adults and presentation as multiple lesions in one sided hemithorax has not been reported. PPB should be considered in the clinical and radiographic differential diagnosis of multicystic lesions, even when the patient is a young adult. Keywords: Blastoma, Malignant,
Pleuropulmonary

$* * * * * * * * *$

Paliwal N, Gupta K, Mullick S, Dewan RK, Katiyar S. Pleuropulmonary blastoma in an adult: A case report. International Journal of Case Reports and Images 2013;4(1):46-50.

$* * * * * * * * *$

doi:10.5348/ijcri-2013-01-257-CR-11

\section{INTRODUCTION}

Pleuropulmonary blastoma (PPB) is a unique dysontogenetic neoplasm of childhood. Its primitive, sarcomatous features are analogous to those of other dysontogenetic tumors such as Wilm's tumor, hepatoblastoma, neuroblastoma, and embryonal rhabdomyosarcoma [1]. PPB was classified into three groups by Dehner et al. in 1995 as cystic (type I), mixed (type II), and solid (type III). Type I has a more favorable prognosis than type II and III [2]. This rare and aggressive neoplasm arises from the lung, pleura, or both and is a disease of the first decade of life [3]. PPB is observed rarely in adults and clinical presentation as in our case has not been reported in literature. There is no optimal defined treatment regimen for adult cases [4]. 
We are reporting a case of PPB in a 30-years-old male presenting with three multicystic lesions in right hemithorax and give a brief review of literature.

\section{CASE REPORT}

A 30-year-old male patient presented with chest pain, dyspnea, and low grade fever for last six months. Two years back he had taken antitubercular drugs therapy. He was a non-smoker with no past history of any malignancy. There was no family history of any malignant lesion in his siblings or parents. Physical examination was unremarkable. His chest X-ray revealed homogenous opacity in right mid and lower zones (Figure 1). Computed tomography (CT) scan of chest showed large, multiloculated collection in right thoracic cage with a few foci of calcification in the septations. Underlying lung was collapsed. Two small loculated collections were also seen in right posterior thoracic cavity (Figure 2). The possibilities of hydatid cyst or benign teratoma were considered based on clinical and radiological presentation.

The patient underwent right thoracotomy. Intraoperatively, a large multicystic mass measuring more than $10 \mathrm{~cm}$ in diameter, adherent to lower lobe and diaphragm was seen. Two masses measuring $5 \mathrm{~cm}$ and $4 \mathrm{~cm}$ in diameter were also present in middle and upper lobe of right lung, respectively. Biopsies from masses were taken and sent to the pathology department.

Complete mobilization of largest mass was not possible, so it could not be excised. As facility of frozen section was not available in our hospital, only imprint smears from biopsies were made. All smears showed similar cytological picture and revealed small groups and singly present round to oval to spindle cells with scanty to moderate cytoplasm. Some of the cells were bipolar (Figure 3A). A few cells showed high N:C ratio, moderate cytoplasm with indistinct outline, vesicular chromatin and prominent nucleoli (Figure $3^{\mathrm{B}}$ ). Cytomorphological features were suggestive of malignancy, however, exact typing of the lesion was not possible.

Grossly, resected tissue pieces were $2.8 \times 2.8 \times 0.7 \mathrm{~cm}$ and $3.5 \times 3.0 \times 2.0 \mathrm{~cm}$ in dimensions, multicystic, greyish white to greyish red with multiple, non-communicating cysts, filled with clear fluid and thin intervening septae along with few glistening, solid white nodules (Figure 4). The tissue was fixed in formalin and processed routinely. Histopathological examination revealed cuboidal to low columnar epithelial lined clefts, cysts and fibroepithelial polyps (Figure 5A). Mesenchymal connective tissue revealed disarray of spindle to elongated cells with mild to moderate atypia (Figure $5^{\mathrm{B}}$ ). Cartilagenous islands were also present and some of them revealed hypercellularity of lacunae and focal overlapping. Occasional focus of small darkly stained angulated nuclei (blastemal-like cells) was also seen (Figure 5C). On immunohistochemistry, the lining epithelial cells of cystic spaces were positive for cytokeratin (Dako-AE1/AE3). The stromal cells revealed diffuse vimentin (Dako-V9) positivity (Figure 5D) and focal desmin (Dako-D33) positivity. A diagnosis of pleuropulmonary blastoma type II was made. One small piece of lung tissue with necrotic foci was also received which revealed a necrotizing granulomatous lesion. This focus was negative for acid-fast bacilli. Patient was

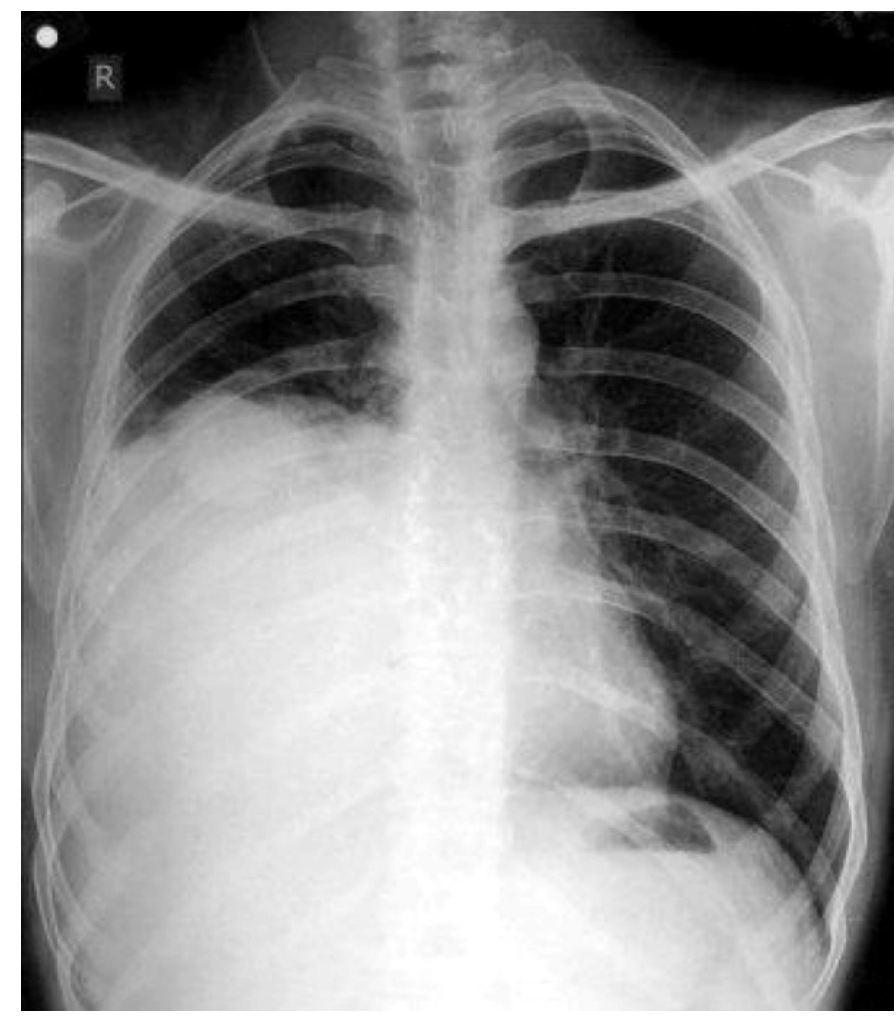

Figure 1: X-ray of chest showing homogenous opacity in right mid and lower zones, silhouetting right heart border and blunted right costophrenic angle (PA view).

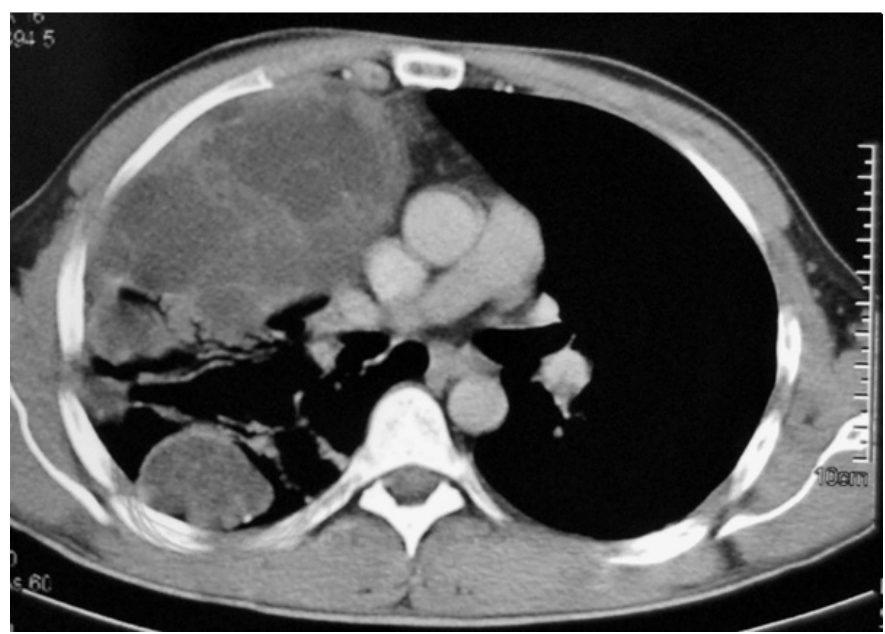

Figure 2: Contrast enhanced computed tomography axial image showing multiple, well-defined, heterogenously enhancing pleural based focal lesions in right hemithorax with enhancing internal septations within the lesions. 


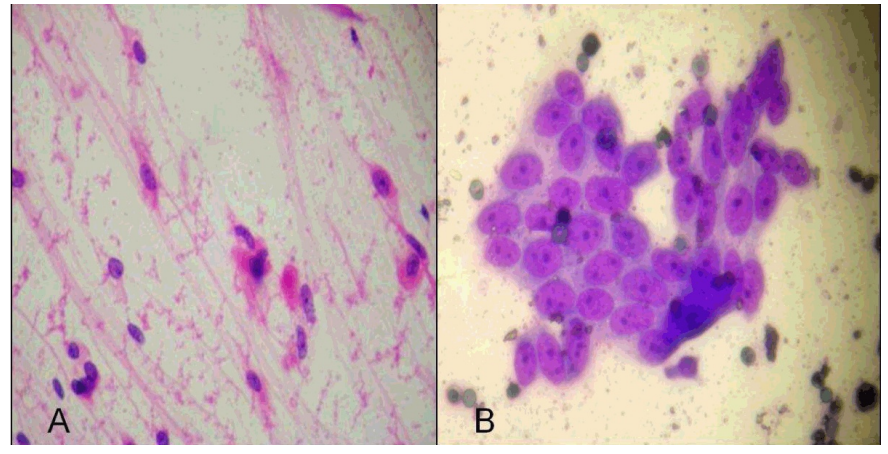

Figure 3: (A) Smear showing round to oval to spindle cells with scanty to moderate cytoplasm (H\&E, x100), (B) Smear showing oval to spindle cells in group with high $\mathrm{N}: \mathrm{C}$ ratio, scanty cytoplasm, vesicular chromatin and prominent nucleoli (Giemsa, x400).

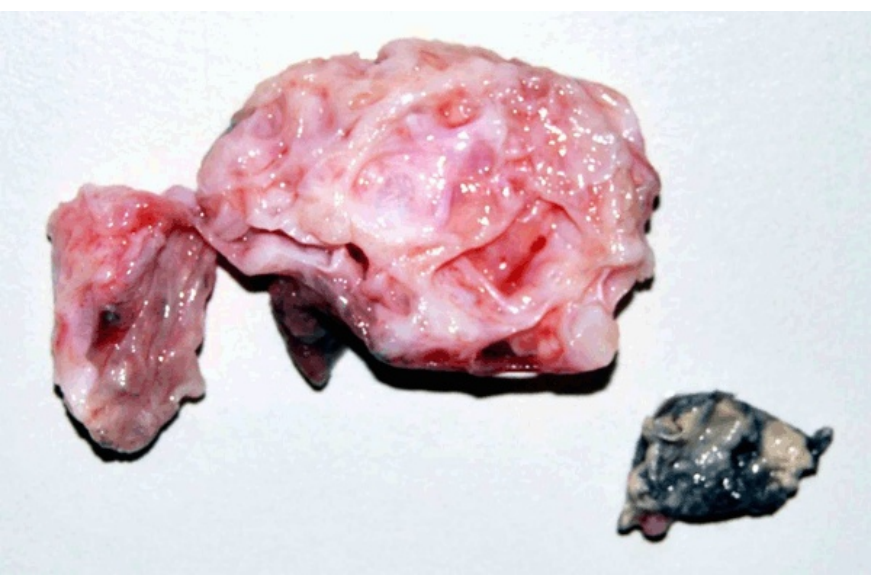

Figure 4: Photomicrograph showing a greyish-red multicystic tissue piece with glistening white nodules and a greyish-black tissue piece (lung tissue) with caseous foci.

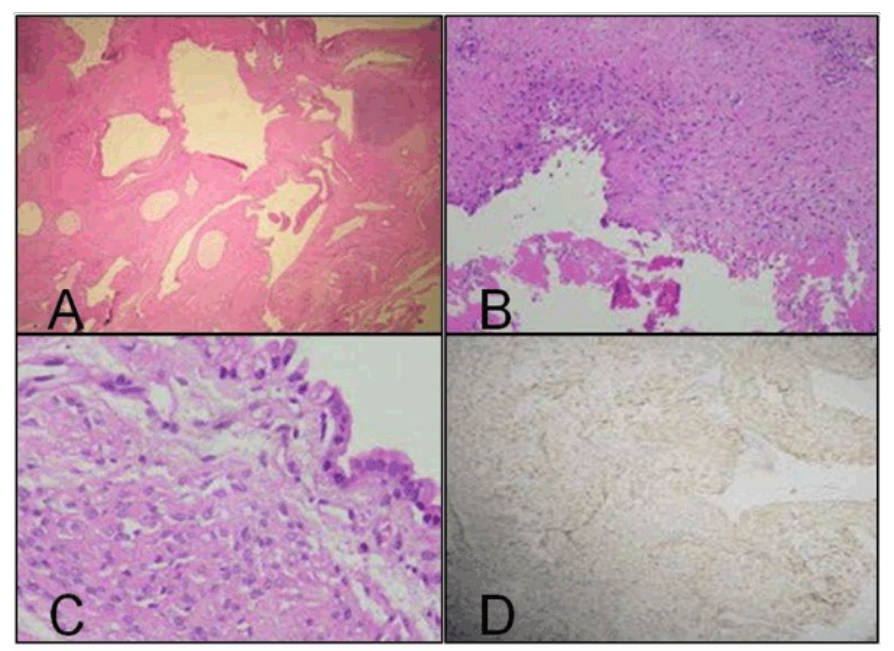

Figure 5: Photomicrographs showing, (A) showing cysts, clefts and cartilaginous island (H\&E, x4O), (B) Cystic space with denuded lining epithelium and subepithelial hypercellular mesenchyme with haphazardly arranged stromal cells (H\&E, x10o), (C) Cystic space lined by cuboidal to low columnar epithelium and subepithelial collection of small round angulated nuclei (H\&E, x400), (D) Diffuse vimentin positivity of stromal cells (Dako-V9;counterstained with H\&E, X100). referred to another hospital for chemotherapy and radiotherapy.

\section{DISCUSSION}

Pleuropulmonary blastoma is a rare and aggressive malignant tumor of childhood. Because PPB has been reported in association with "pre-existing" anomalies of the lung, such as cystic adenomatoid malformation, extralobar sequestration, and bronchogenic cyst, it has been suggested that the foundation for neoplastic transformation is laid during development [1]. Some authors suggest familial disposition and association of PPB tumors with other childhood malignancies [3]. Despite its aggressive growth, PPB causes only a few mild complaints. Often presenting symptoms are fever, cough, hemoptysis, dyspnea and chest pain. The clinical picture of PPB in an adult resembles that in children [5]. In our case also, the patient presented with cough, dyspnea and fever.

About 50\% of all PPB patients develop local recurrence and metastases after intial surgical treatment [6]. Common localizations are brain and spinal cord (44\%), bone (24\%), liver (4\%), and locoregional (76\%). Bilateral manifestations have been described as well [7], but presentation as multiple lesions within one sided hemithorax in an adult has not been reported till date. Atypical presentations such as ruptured cystic teratoma, pleurorrhea and pancreatic metastasis have been reported in literature $[6,8,9]$. The clinical and radiographic differential diagnosis include fungal lesions, congenital cystic adenomatoid malformation, bronchogenic cyst and postinfectious pneumatocele.

Pathologically, the tumor tissue is composed of primitive blastemal cells with focal areas of rhabdomyosarcomatous, chondrosarcomatous and liposarcomatous differentiation. Unlike pulmonary blastoma in adults, the epithelium is never malignant [3]. In our case also, blastemal cell collection was seen with normal appearing epithelium but no well defined sarcomatous component was noted. Sheets and nodules of blastemal-type cells, cystic areas and myxoid change in stroma seen in our case are also described by Hill et al. [1]. Three pathologic types of PPB, based on gross and microscopic features have been defined: type I is a purely cystic lesion that may be mistaken clinically and pathologically for a congenital lung cyst, type II is a cystic and solid lesion with areas of thickening and/or nodularity with or without a relationship to the cysts, and type III is a purely solid tumor consisting of friable, gelatinous to mucoid, lobulated tissue often accompanied by hemorrhage and necrosis [1]. In non-cystic cases, without an overt rhabdomyoblastic component, entities such as monophasic synovial sarcoma, malignant teratoma, and undifferentiated sarcoma should be considered. Immunohistochemistry plays an important role in differentiating between PPB such entitles. In immunohistochemical studies; the most common findings are vimentin positivity and S-100 protein 
positivity in cartilagenous foci and desmin positivity in areas of rhabdomyoblastic differentiation. The only typical characteristic of PPB is vimentin positivity [4]. In cytogenetic studies of childhood cases, chromosomal anomalies of trisomies 8 and 2 have been detected. But as studied by Gonullu et al., in adult patients, interestingly, karyotypic abnormalities such as trisomies 8 and 2 were not demonstrated [4]. Germline mutations in DICER1 has also been described in cases of familial PPB and ovarian sex cord-stromal tumors [10]. We were not provided with the opportunity to perform cytogenetic studies in the current case.

While tumor size $>5 \mathrm{~cm}$ and mediastinal and/or pleural invasion are poor prognostic factors, the most important prognostic factor is the total excision of the mass with clear margins. Postoperative radiotherapy is recommended in cases with incomplete resection [11]. Cyclophosphamide, doxorubicin, ifosfamide, etoposide, vincristine are commonly used agents in the treatment of PPB. The most common combination is vincristine, dactinomycin, cyclophosphamide (VAC) regimen [4]. Therapy should include surgical tumor resection and subsequent chemotherapy and radiotherapy. In a review of 50 cases of PPB in children by Priest et al., overall survival for all patients was $63 \%$ after two years and $45 \%$ after five years. Median survival was calculated and it was 15.5 months from diagnosis and 5.5 months from recurrence [6].

\section{CONCLUSION}

Pleuropulmonary blastoma is very rare tumor in adults and its presentation as multiple lesions in one sided hemithorax has not been reported. Pleuropulmonary blastoma should be considered in the clinical and radiographic differential diagnosis when hydatid cyst or benign teratoma is suspected in view of its multicystic appearance, even when the patient is a young adult.

$* * * * * * * * *$

\section{Author Contributions}

Nidhi Paliwal - Substantial contributions to conception and design, Acquisition of data, Analysis and interpretation of data, Drafting the article, Revising it critically for important intellectual content, Final approval of the version to be published

Kumud Gupta - Substantial contributions to conception and design, Acquisition of data, Analysis and interpretation of data, Drafting the article, Revising it critically for important intellectual content, Final approval of the version to be published

Shalini Mullick - Substantial contributions to conception and design, Acquisition of data, Analysis and interpretation of data, Drafting the article, Revising it critically for important intellectual content, Final approval of the version to be published

Ravindra K Dewan - Substantial contributions to conception and design, Acquisition of data, Analysis and interpretation of data, Drafting the article, Revising it critically for important intellectual content, Final approval of the version to be published

Sandeep Katiyar - Substantial contributions to conception and design, Acquisition of data, Analysis and interpretation of data, Drafting the article, Revising it critically for important intellectual content, Final approval of the version to be published

\section{Guarantor}

The corresponding author is the guarantor of submission.

\section{Conflict of Interest}

Authors declare no conflict of interest.

\section{Copyright}

(C) Nidhi Paliwal et al. 2013; This article is distributed under the terms of Creative Commons Attribution 3.0 License which permits unrestricted use, distribution and reproduction in any means provided the original authors and original publisher are properly credited. (Please see www.ijcasereportsandimages.com/copyright-policy.php for more information.)

\section{REFERENCES}

1. Hill DA, Sadeghi S, Schultz MZ, Burr JS, Dehner LP. Pleuropulmonary blastoma in an adult: An initial case report. Cancer 1999;85(11):2368-74.

2. Dehner LP, Watterson J, Priest J. Pleuropulmonary blastoma: a unique intrathroacic-pulmonary neoplasm of childhood. Perspect pediatr pathol 1995;18:214-26.

3. Manivel J, Priest J, Watterson J, et al. Pleuropulmonary blastoma. The so-called pulmonary blastoma of childhood. cancer 1988;62(8):1516-26.

4. Gonullu G, Evrense T, Kurt E, et al. Pleuropulmonary blastoma in an adult patient: Report of a case. Turkish journal of cancer 2007;37(4):158-61.

5. Walles T, Teebken OE, Bartels M, et al. Pancreatic metastasis of a pleuropulmonary blastoma in an adult. Annals of oncology 2000;11(12):1609-11.

6. Priest JR, McDermott MB, Bhatia S, Watterson J, Manivel JC, Dehner LP. Pleuropulmonary blastoma. A clinicopathologic study of 50 cases. Cancer 1997;80(1):147-61.

7. Lallier M, Bouchard S, Di Lorenzo M, et al. Pleuropulmonary blastoma: A rare pathology with an even rarer presentation. $J$ Pediatr Surg 1999;34(7):1057-9.

8. Lee CH, Kim KI, Kim YD, et al. Pleuropulmonary blastoma in a young adult presenting as a ruptured cystic teratoma in radiology. J Korean Med Sci 2003 Aug;18(4):595-8.

9. Liu AH, Zheng WY, WU L. [Pleuropulmonary blastoma in an adult women with pleurorrhea as the major clinical manifestation: report of a case]. Nan Fang Yi Ke Da Xue Xue Bao 2008 Dec;28(12):2241-3.

10. Schultz KAP, Pacheco MC, Yang J, et al. Ovarian sex cord -stromal tumors, pleuropulmonary blastoma and DICER1 mutations: A report from the 
International Pleuropulmonary blastoma Registry. Gynecol Oncol 2011 Aug;122(2):246-50.

11. Parsons SK, Fishman SJ, Hoorntje LE, et al. Aggressive multimodal treatment of
Pleuropulmonary blastoma. Ann Thorac Surg 2001;72(3):939-42.
Access full text article on other devices

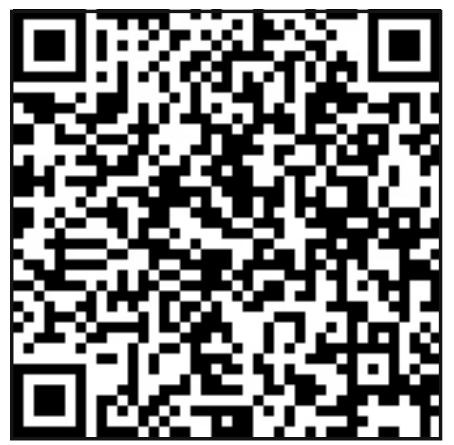

Access PDF of article on other devices

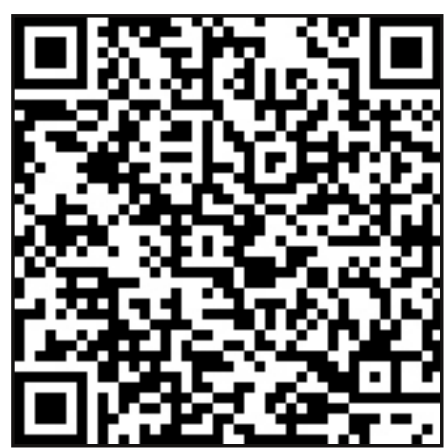

